\title{
Coping mediates the influence of personality on life satisfaction in patients with rheumatic diseases
}

\author{
Manja Vollmann $^{1,2} \cdot$ Jörg Pukrop $^{3} \cdot$ Christel Salewski $^{1}$
}

Received: 8 January 2016/Revised: 15 February 2016/Accepted: 15 February 2016/Published online: 22 February 2016

(C) International League of Associations for Rheumatology (ILAR) 2016

\begin{abstract}
A rheumatic disease can severely impair a person's quality of life. The degree of impairment, however, is not closely related to objective indicators of disease severity. This study investigated the influence and the interplay of core psychological factors, i.e., personality and coping, on life satisfaction in patients with rheumatic diseases. Particularly, it was tested whether coping mediates the effects of personality on life satisfaction. In a cross-sectional design, 158 patients diagnosed with a rheumatic disease completed questionnaires assessing the Big 5 personality traits (BFI-10), several disease-related coping strategies (EFK) and life satisfaction (HSWBS). Data were analyzed using a complex multiple mediation analysis with the Big 5 personality traits as predictors, coping strategies as mediators and life satisfaction as outcome. All personality traits and seven of the nine coping strategies were associated with life satisfaction $(r \mathrm{~s}>|0.16|, p \mathrm{~s} \leq 0.05)$. The mediation analysis revealed that personality traits had no direct, but rather indirect effects on life satisfaction through coping. Neuroticism had a negative indirect effect on life satisfaction through less active problem solving and more depressive coping (indirect effects $>-0.03, p \mathrm{~s}<0.05$ ). Extraversion, agreeableness, and conscientiousness had positive indirect effects on life satisfaction through more active
\end{abstract}

Manja Vollmann

manja.vollmann@fernuni-hagen.de

1 Department of Health Psychology, University of Hagen, Hagen, Germany

2 Department of Clinical and Health Psychology, Utrecht University, Utrecht, The Netherlands

3 Department of Mathematics and Computer Science, University of Bremen, Bremen, Germany problem solving, less depressive coping and/or a more active search for social support (indirect effects $>0.06, p s<0.05$ ). Personality and coping play a role in adjustment to rheumatic diseases. The interplay of these variables should be considered in psychological interventions for patients with rheumatic diseases.

Keywords Big 5 personality traits · Coping $\cdot$ Life satisfaction $\cdot$ Mediation $\cdot$ Rheumatic diseases

\section{Introduction}

According to the Global Burden of Diseases 2013 Study [1], rheumatic or musculoskeletal disorders are among the leading causes of years lived with disability. Rheumatic diseases are chronic conditions affecting joints, bones, cartilage, tendons, ligaments, and muscles. Usually, rheumatic diseases are progressive and characterized by pain, deformity, stiffness, and swelling in or around one or more joints [2]. Rheumatic diseases not only cause physical impairment but can also severely impair a person's psychological wellbeing [3, 4]. Objective indicators of disease severity, such as inflammatory activity and number of swollen joints, are not closely related to psychological impairment $[5,6]$. This suggests that psychological factors play a role in adjustment to rheumatic diseases.

Important contributors to psychological adjustment to chronic diseases are personality and coping [7, 8]. Bolger and Zuckerman [9] propose different models for the interplay of these two psychological factors in affecting outcomes. Their differential choice model (a mediation model) implies that personality determines the choice of coping strategies, which in turn have an impact on outcomes. Previous research findings lend some support to the notion that personality and 
coping play a role in adjustment to rheumatic diseases [10-15].

Several systematic reviews report associations between coping and indicators of psychological adjustment in patients with rheumatic diseases. Avoidant coping strategies, such as wishful thinking and resting, and emotion-focused coping strategies, such as catastrophizing and venting emotions, are consistently related to increased psychological distress and negative mood. Also, approach coping strategies and problem-focused coping strategies are related to psychological outcomes such as distress and pain. However, the pattern is less consistent [10-12].

Studies examining the impact of personality on adjustment to rheumatic diseases have mainly focused on the Big 5 personality traits neuroticism and extraversion. Results indicate that neuroticism is associated with higher and extraversion with lower levels of psychological distress and that these associations are partly mediated by coping [13-15].

Studies investigating the impact of other personality traits than neuroticism and extraversion on adjustment to rheumatic diseases are rare, although studies certify the other Big 5 personality traits, i.e., openness for experience, agreeableness, and conscientiousness, a substantial relevance with regard to subjective well-being [16]. Also, previous studies examining the mediating effect of coping on the relationship between personality and health outcomes in patients with rheumatic diseases only included a limited number of coping strategies.

Therefore, the aim of the present study was to extend previous research by considering all Big 5 personality traits and a wide range of disease-related coping strategies as potential predictors of adjustment to rheumatic diseases. Based on the differential choice model [9], the mediating role of coping in the relationship between personality and life satisfaction in patients with rheumatic diseases was investigated. Statistical analyses that allow to simultaneously estimate the effects of all personality traits through all coping strategies were used [17], with the advantage of revealing unique effects (see Fig. 1). The results may be of practical interest in the context of psychological interventions aiming at promoting the adjustment to rheumatic diseases.

\section{Method}

\section{Procedure and participants}

Participants were recruited in the Clinic of Rheumatology of the Red Cross Hospital Bremen, Germany. Patients diagnosed with a rheumatic disease received information about the study and were invited to participate. Participation was voluntary and anonymous. The study was approved by the Ethical Committee of the Bremen State Chamber of Physicians.
A total of 158 patients ( $68 \%$ female) with a mean age of 55.79 years $(S D=13.44)$ returned the completed questionnaires. Included patients suffered from their rheumatic disease, i.e., rheumatoid arthritis, osteoarthritis, or soft-tissue rheumatism, for an average of 11.84 years $(S D=9.79)$.

\section{Measures}

Personality The German version of the Big Five Inventory-10 (BFI-10) [18] was used to measure the Big 5 personality traits neuroticism, extraversion, openness for experience, agreeableness, and conscientiousness with two items each $(0.58 \leq r \mathrm{~s} \leq 0.80)$.

Disease-related coping The Essener Coping Questionnaire (EFK) [19] was used to assess coping with rheumatic diseases. The EFK measures the strategies active problem solving, distraction and encouragement, information seeking, minimization and wishful thinking, depressive coping, accepting help, active search for social support, confidence in doctor's competence, and religious coping with five items each $(0.59 \leq \alpha \leq 0.79)$. The questionnaire has been used and validated in patients with various chronic diseases.

Life satisfaction Life satisfaction was measured with the subscale general satisfaction with life of the Habitual Subjective Well-Being Scale (HSWBS) [20] which contains seven items $(\alpha=0.91)$.

\section{Data analyses}

First, bivariate correlations between the study variables were calculated. Second, a multiple mediation analysis using the MEDIATE-macro [17] with the Big 5 personality traits as predictors, the coping strategies as mediators, and life satisfaction as outcome was conducted. As finding mediation effects is less likely when predictor and potential mediator are unrelated, only coping strategies that correlated significantly with at least one Big 5 personality trait were included in the analysis. The mediation analysis consisted of two parts: First, total and direct effects of each Big 5 personality trait on life satisfaction were estimated by a stepwise regression analysis in which personality traits were entered in the first and coping strategies in the second step. Second, indirect effects of each Big 5 personality trait on life satisfaction via each coping strategy were estimated by bootstrapping. Five thousand bootstrap samples were used and $95 \%$ bias corrected and accelerated confidence intervals were calculated. As recommended by Hayes and Preacher [17], unstandardized coefficients will be reported. 
Fig. 1 Theoretical mediation model with the Big 5 personality traits as predictors, coping strategies as mediators and life satisfaction as outcome. Variables in bold boxes were included in the final multiple mediation analysis

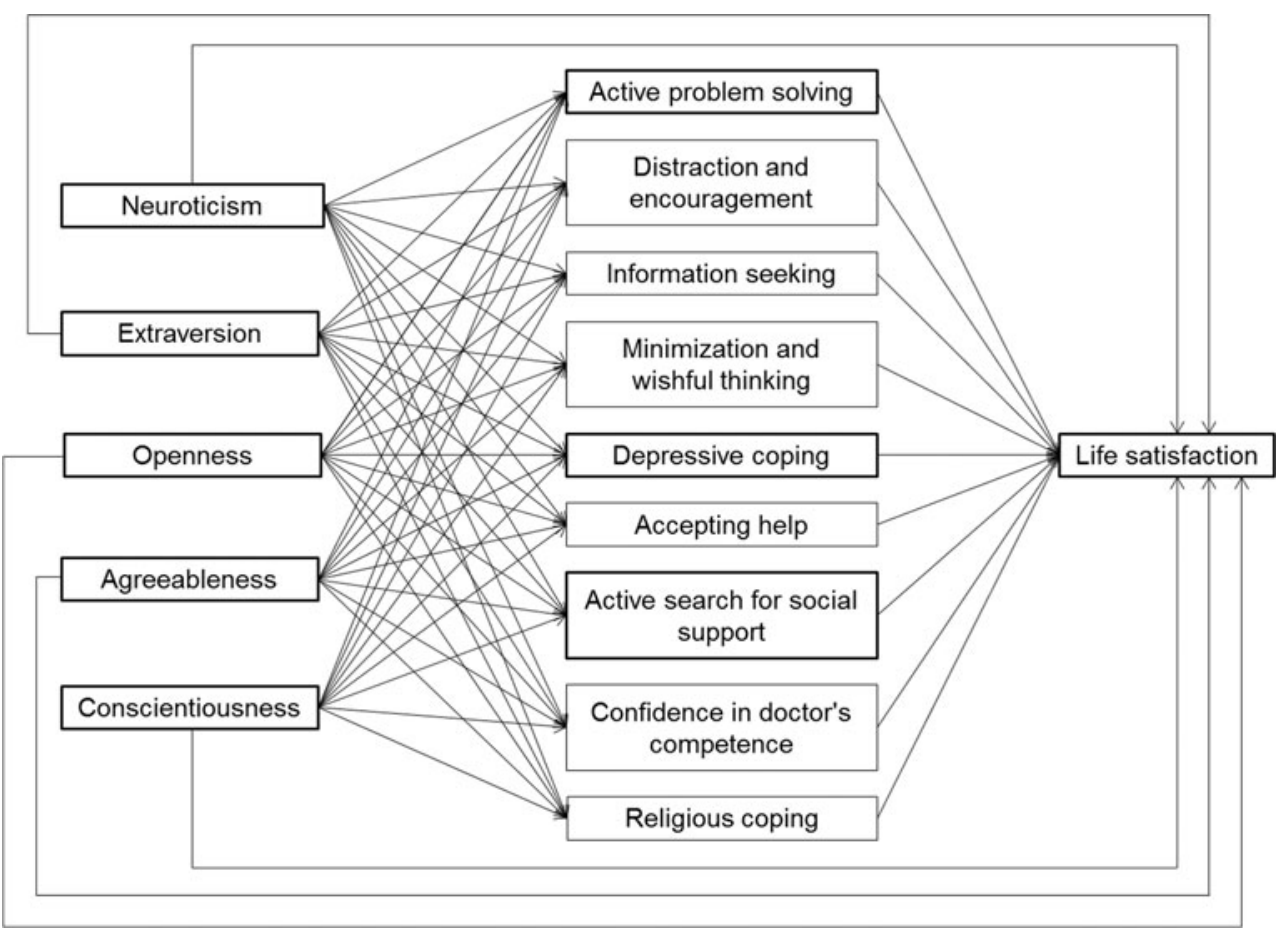

\section{Results}

\section{Correlation analysis}

Correlation analyses (see Table 1) revealed that (1) all Big 5 personality traits were significantly related to life satisfaction, (2) the coping strategies active problem solving, distraction and encouragement, depressive coping, accepting help, active search for social support, confidence in doctor's competence, and religious coping were significantly correlated with life satisfaction, and (3) only the coping strategies active problem solving, depressive coping, and active search for social support were significantly associated with at least one Big 5 personality trait.

\section{Mediation analysis}

The multiple mediation analysis was conducted with the Big 5 personality traits as predictors, the coping strategies active problem solving, depressive coping, and active search for social support as mediators, and life satisfaction as outcome (see also Fig. 1).

The regression analysis (see Table 2) revealed in step $1 \mathrm{a}$ significant negative total effect of neuroticism and a significant positive total effect of extraversion on life satisfaction, indicating that neuroticism was related to lower and extraversion to higher levels of life satisfaction. In step 2 of the regression analysis, none of the personality traits had a significant direct effect on life satisfaction, while all three coping

Table 1 Results of the correlation analysis: correlations between the Big 5 personality traits, coping strategies, and life satisfaction

\begin{tabular}{|c|c|c|c|c|c|c|}
\hline & Neuroticism & Extraversion & Openness for experience & Agreeableness & Conscientiousness & Life satisfaction \\
\hline Active problem solving & $-0.23 * *$ & $0.22 * *$ & $0.19 *$ & 0.10 & $0.39 * * *$ & $0.38 * * *$ \\
\hline Distraction and encouragement & -0.06 & 0.11 & 0.05 & 0.06 & 0.01 & $0.34 * * *$ \\
\hline Information seeking & 0.11 & 0.01 & -0.02 & 0.08 & 0.02 & -0.09 \\
\hline Minimization and wishful thinking & 0.12 & -0.15 & -0.08 & 0.03 & -0.08 & 0.09 \\
\hline Depressive coping & $0.33 * * *$ & $-0.38 * * *$ & $-0.18^{*}$ & -0.14 & $-0.20 * *$ & $-0.41 * * *$ \\
\hline Accepting help & 0.02 & 0.15 & -0.01 & 0.11 & 0.01 & $0.16^{*}$ \\
\hline Active search for social support & $-0.16^{*}$ & $0.41 * * *$ & $0.20^{*}$ & $0.26^{* *}$ & $0.19^{*}$ & $0.44 * * *$ \\
\hline Confidence in doctor's competence & -0.06 & 0.12 & 0.07 & 0.07 & 0.12 & $0.18^{*}$ \\
\hline Religious coping & -0.11 & 0.06 & 0.07 & $0.18^{*}$ & 0.10 & $0.24 * *$ \\
\hline Life satisfaction & $-0.23 * *$ & $0.37 * * *$ & $0.17^{*}$ & $0.16^{*}$ & $0.21 * *$ & \\
\hline
\end{tabular}

$* * * p<0.001, * * p<0.01, * p \leq 0.05$ 
Table 2 Results of the stepwise regression analysis with life satisfaction as outcome: Total and direct effects of the Big 5 personality traits on life satisfaction

\begin{tabular}{lcc}
\hline Predictors & B step 1 & B step 2 \\
\hline Step 1: adj. $R^{2}=0.13, F(5,152)=5.71^{* * *}$ & & \\
Neuroticism & $-0.19^{*}$ & -0.06 \\
Extraversion & $0.26^{* *}$ & 0.06 \\
Openness for experience & 0.10 & 0.02 \\
Agreeableness & 0.11 & 0.02 \\
Conscientiousness & 0.10 & 0.00 \\
Step 2: $\Delta R^{2}=0.17, F(3149)=12.92^{* * *} ;$ adj. $R^{2}=0.30$, & \\
$F(8,149)=9.26^{* * *}$ & & \\
Active problem solving & & $0.24^{*}$ \\
Depressive coping & & $-0.40^{* * *}$ \\
Active search for social support & & $0.38^{* * *}$ \\
\hline
\end{tabular}

B's in step 1 represent total effects of the Big 5 personality traits on life satisfaction. B's in step 2 represent direct effects of the Big 5 personality traits on life satisfaction. Unstandardized coefficients are reported

*** $p<0.001, * * p<0.01, * p<0.05$

strategies were significantly associated with the outcome. Active problem solving and active search for social support were positively related to life satisfaction, while depressive coping showed a negative effect on life satisfaction. Thirty percent of the variance in life satisfaction could be explained.

The bootstrap procedure revealed several indirect effects. Neuroticism had a negative indirect effect on life satisfaction via active problem solving, -0.03 , BCa $95 \%$ CI $(-0.100$, $-0.001)$, and depressive coping, $-0.08 \mathrm{BCa} 95 \% \mathrm{CI}$ $(-0.150,-0.030)$. This indicates that neuroticism was related to less active problem solving and more depressive coping, which in turn were related to lower life satisfaction. Extraversion had a positive indirect effect on life satisfaction via depressive coping, 0.08 , BCa $95 \% \mathrm{CI}(0.030,0.171)$, and active search for social support, 0.10, BCa $95 \%$ CI (0.047, 0.181 ). Thus, extraversion was related to less depressive coping and more active search for social support, which in turn were related to higher life satisfaction. Agreeableness and conscientiousness had positive indirect effects on life satisfaction through more active search for social support, $0.06, \mathrm{BCa}$ $95 \% \mathrm{CI}(0.006,0.155)$, and more active problem solving, 0.08 , BCa $95 \%$ CI $(0.018,0.215)$, respectively. In detail, agreeableness was related to more active search for social support and conscientiousness was related to more active problem solving, which in turn were related to higher life satisfaction.

\section{Discussion}

The present findings confirm the assumption that both personality and coping play a role in adjustment to rheumatic diseases. All Big 5 personality traits and nearly all coping strategies showed univariate associations with life satisfaction. With regard to personality, the results are in line with previous studies investigating neuroticism and extraversion in patients with rheumatic diseases [13-15], and with the assumption that openness to experience, agreeableness, and conscientiousness are also related to adjustment to rheumatic diseases. Turning to coping, the findings support previous research in patients with rheumatic diseases [10-12] and emphasize the relevance of coping in adjustment to rheumatic diseases.

Regarding the interplay of personality and coping in affecting adjustment to rheumatic diseases, the present results support the differential choice model that proposes that coping serves as a mediator of the relationship between personality and outcomes [9]. In the present study, no direct, but several indirect effects of the Big 5 personality traits on life satisfaction were found. Neuroticism is related to lower life satisfactions via more depressive coping and less active problem solving. Extraversion, agreeableness, and conscientiousness positively affect life satisfaction via more active problem solving, less depressive coping, and/or more active search for social support. This conforms to previous studies that found the effects of neuroticism and extraversion to be mediated by coping $[14,15]$ and extends this knowledge to two more Big 5 personality traits. Only for openness for experience no mediating effects could be found, probably pointing to the somewhat different nature of this personality dimension [21]. The results also demonstrate the advantage of applying more complex statistical methods, such as multiple mediation analysis, to reveal structures underlying the relations between personality, coping, and life satisfaction that would otherwise remain concealed.

These findings are of practical interest as they indicate that personality should be considered in psychological interventions aiming to promote adjustment to rheumatic diseases. The improvement of coping skills is typically included in such interventions [22, 23], and significant intervention effects regarding changes in coping behavior have been reported [24, 25]. However, according to our findings, the use of some coping strategies is determined by personality traits, which suggests that a patient's personality might modulate the effectiveness of an intervention to change coping behavior. High levels of neuroticism, for example, might limit the increase in active problem solving that can be achieved through an intervention. On the other hand, a patient's personality could also serve as a basis for tailored interventions that focus on the change of coping strategies inherent to a certain personality profile. Patients with low levels of extraversion and agreeableness, for example, will probably profit most from an intervention targeting depressive coping and facilitating an active search for social support. Future studies investigating the effects of psychological interventions in patients with rheumatic diseases are well advised to explicitly consider personality as a 
modulating factor. Certainly, if depressive coping and low life satisfaction are indicators of an underlying depressive disorder, this should be treated in the first place.

Some limitations need to be acknowledged. Due to the cross-sectional design, the direction of causality in the associations between the constructs could not definitely be determined. Other mediation models are theoretically less plausible, but still possible. Longitudinal studies are needed to address this issue. Generic questionnaires were used to assess disease-related coping and life satisfaction. Further research would profit from measuring coping strategies and psychological outcomes with questionnaires explicitly designed for patients suffering from rheumatic diseases. The sample consisted of patients with various rheumatic diseases. To get a deeper insight in disease-specific processes, future studies should focus on more homogeneous samples. Acknowledging these limitations, this study contributes to the understanding of determinants of adjustment to rheumatic diseases.

Compliance with ethical standards Participation was voluntary and anonymous. The study was approved by the Ethical Committee of the Bremen State Chamber of Physicians.

Disclosures None

Funding No funding was received for this study.

\section{References}

1. Global Burden of Disease Study 2013 Collaborators (2015) Global, regional, and national incidence, prevalence, and years lived with disability for 301 acute and chronic diseases and injuries in 188 countries, 1990-2013: a systematic analysis for the Global Burden of Disease Study 2013. Lancet 386:743-800. doi:10. 1016/s0140-6736(15)60692-4

2. WHO Chronic Rheumatic Conditions, Fact Sheet, WHO, Geneva, available at: www.who.int/chp/topics/rheumatic/en/

3. Patten SB, Williams JV, Wang J (2006) Mental disorders in a population sample with musculoskeletal disorders. BMC Musculoskel Dis 7:37-46. doi:10.1186/1471-2474-7-37

4. Geenen R, Newman S, Bossema ER, Vriezekolk JE, Boelen PA (2012) Psychological interventions for patients with rheumatic diseases and anxiety or depression. Best Pract Res Cl Rh 26:305-319. doi:10.1016/j.berh.2012.05.004

5. Nyklíček I, Hoogwegt F, Westgeest T (2015) Psychological distress across twelve months in patients with rheumatoid arthritis: the role of disease activity, disability, and mindfulness. J Psychosom Res 78:162-167. doi:10.1016/j.jpsychores.2014.08.004

6. Pollard LC, Choy EH, Gonzalez J, Khoshaba B, Scott DL (2006) Fatigue in rheumatoid arthritis reflects pain, not disease activity. Rheumatology 45:885-889. doi:10.1093/rheumatology/kel021

7. Stanton AL, Revenson TA, Tennen H (2007) Health psychology: psychological adjustment to chronic disease. Annu Rev Psychol 58: 565-592. doi:10.1146/annurev.psych.58.110405.085615

8. de Ridder D, Geenen R, Kuijer R, van Middendorp H (2008) Psychological adjustment to chronic disease. Lancet 372:246255. doi:10.1016/s0140-6736(08)61078-8
9. Bolger N, Zuckerman A (1995) A framework for studying personality in the stress process. J Pers Soc Psychol 69:890-902. doi:10. 1037/0022-3514.69.5.890

10. Benyon K, Hill S, Zadurian N, Mallen C (2010) Coping strategies and self-efficacy as predictors of outcome in osteoarthritis: a systematic review. Musculoskelet Care 8:224-236. doi:10.1002/msc.187

11. Ramjeet J, Smith J, Adams M (2008) The relationship between coping and psychological and physical adjustment in rheumatoid arthritis: a literature review. J Clin Nurs 17:418-428. doi:10.1111/j. 1365-2702.2008.02579.x

12. Vriezekolk JE, van Lankveld WGJM, Geenen R, van den Ende CHM (2011) Longitudinal association between coping and psychological distress in rheumatoid arthritis: a systematic review. Ann Rheum Dis 70:1243-1250. doi:10.1136/ard.2010.143271

13. Benka J, Nagyova I, Rosenberger J, Macejova Z, Lazurova I, van der Klink J et al (2014) Is coping self-efficacy related to psychological distress in early and established rheumatoid arthritis patients? J Dev Phys Disabil 26:285-297. doi:10.1007/s10882-013-9364-y

14. Persson LO, Sahlberg D (2002) The influence of negative illness cognitions and neuroticism on subjective symptoms and mood in rheumatoid arthritis. Ann Rheum Dis 61:1000-1006

15. Suurmeijer TPBM, van Sonderen FLP, Krol B, Doeglas DM, van den Heuvel WJA, Sanderman R (2005) The relationship between personality, supportive transactions and support satisfaction, and mental health of patients with early rheumatoid arthritis. Results from the Dutch part of the EURIDISS study. Soc Indic Res 73: 179-197. doi:10.1007/s11205-004-0562-y

16. Steel P, Schmidt J, Shultz J (2008) Refining the relationship between personality and subjective well-being. Psychol Bull 134: 138-161. doi:10.1037/0033-2909.134.1.138

17. Hayes AF, Preacher KJ (2014) Statistical mediation analysis with a multicategorical independent variable. Br J Math Stat Psychol 67: 451-470. doi:10.1111/bmsp. 12028

18. Rammstedt B, John OP (2007) Measuring personality in one minute or less: a 10-item short version of the Big Five Inventory in English and German. J Res Pers 41:203-212. doi:10.1016/j.jrp.2006.02.001

19. Franke GH, Mähner N, Reimer J, Spangemacher B, Esser J (2000) Erste Überprüfung des Essener Fragebogens zur Krankheitsverarbeitung (EFK) an sehbeeinträchtigten Patienten [First evaluation of the Essener Coping Questionnaire (EFK) in patients with visual impairment]. J Individ Differ 21:166-172

20. Dalbert C (1992) Subjektives Wohlbefinden junger Erwachsener: theoretische und empirische Analysen der Struktur und Stabilität [Subjective well-being of young adults: theoretical and empirical analyses of structure and stability]. J Individ Differ 13:207-220

21. Ferguson E, Patterson F (1998) The five factor model of personality: openness a distinct but related construct. Pers Indiv Differ 24: 789-796. doi:10.1016/S0191-8869(97)00241-9

22. Vriezekolk JE, Geenen R, van den Ende CHM, Slot H, van Lankveld WGJM, van Helmond T (2012) Behavior change, acceptance, and coping flexibility in highly distressed patients with rheumatic diseases: feasibility of a cognitive-behavioral therapy in multimodal rehabilitation. Patient Educ Couns 87:171-177. doi:10.1016/j.pec. 2011.09.001

23. Knittle K, Maes S, de Gucht V (2010) Psychological interventions for rheumatoid arthritis: examining the role of self-regulation with a systematic review and meta-analysis of randomized controlled trials. Arthritis Care Res 62:1460-1472. doi:10.1002/acr.20251

24. Astin JA, Beckner W, Soeken K, Hochberg MC, Berman B (2002) Psychological interventions for rheumatoid arthritis: a metaanalysis of randomized controlled trials. Arthritis Care Res 47: 291-302. doi:10.1002/art.10416

25. Shin SY, Kolanowski AM (2010) Best evidence of psychosocially focused nonpharmacologic therapies for symptom management in older adults with osteoarthritis. Pain Manag Nurs 11:234-244. doi: 10.1016/j.pmn.2009.06.001 